\title{
Planar two-loop five-gluon amplitudes from numerical unitarity
}

\author{
S. Abreu, ${ }^{1}$ F. Febres Cordero, ${ }^{1}$ H. Ita, ${ }^{1}$ B. Page,${ }^{1}$ and M. Zeng ${ }^{2}$ \\ ${ }^{1}$ Physikalisches Institut, Albert-Ludwigs-Universität Freiburg, D-79104 Freiburg, Germany \\ ${ }^{2}$ Mani L. Bhaumik Institute for Theoretical Physics, \\ UCLA Department of Physics and Astronomy Los Angeles, California 90095, USA
}

(Received 10 January 2018; published 20 June 2018)

\begin{abstract}
We present a calculation of the planar two-loop five-gluon amplitudes. The amplitudes are obtained in a variant of the generalized unitarity approach suitable for numerical computations, which we extend for use with finite field arithmetics. Employing a new method for the generation of unitarity-compatible integration-by-parts identities, all helicity amplitudes are reduced to a linear combination of master integrals for the first time. The approach allows us to compute exact values for the integral coefficients at rational phase-space points. All required master integrals are known analytically, and we obtain arbitraryprecision values for the amplitudes.
\end{abstract}

DOI: $10.1103 /$ PhysRevD.97.116014

\section{INTRODUCTION}

The expected precision of upcoming cross-section measurements at the Large Hadron Collider at CERN currently drives the development of new computational methods in perturbative quantum-field theory and, in particular, in QCD. At the same time, formal advances in the field of amplitudes are finding more and more traction and are helping to devise more efficient approaches to multiloop computations. Here, we aim to contribute to these developments by refining and extending methods to compute phenomenologically relevant two-loop scattering amplitudes. With our results, we advance the state of the art in the calculation of loop amplitudes with many external particles. Besides their importance for phenomenology, the new results highlight the potential of the methods we employ.

In this paper, we present the computation of two-loop gluonic amplitudes which contribute to three-jet production at hadron colliders at next-to-next-to-leading order (NNLO). Predictions for these processes can be used for constraining the strong coupling, as it can be extracted from precision measurements of three- to two-jet-production ratios (see e.g., Ref. [1,2]). While the finite parts of our results can be integrated over phase space, significant additional developments will be required to obtain the full NNLO predictions. Probably the most challenging step will be the consistent combination of all virtual and realradiation contributions to NNLO accuracy. Nevertheless,

Published by the American Physical Society under the terms of the Creative Commons Attribution 4.0 International license. Further distribution of this work must maintain attribution to the author(s) and the published article's title, journal citation, and DOI. Funded by SCOAP ${ }^{3}$. we are optimistic that by providing key ingredients towards this goal, such as the two-loop scattering amplitudes, we will spur progress in the field to obtain NNLO predictions for three-jet production in the coming years.

Currently, only a limited set of amplitudes with five or more external particles are known to two-loop order in QCD. First benchmark results for two-loop five-gluon amplitudes were obtained for the all-plus-helicity configuration [3,4], whose computation relied on compact analytic expressions for the integrands. These were computed with the unitarity method [5-8], coupled with efficient integrand-reduction techniques [9-13]. The final amplitudes were first obtained by integrating the optimized integrands numerically and later by explicit reduction to master integrals [14]. The relevant master integrals have been independently evaluated in Ref. [15]. More recently, the all-plus two-loop amplitudes were obtained from a recursive approach [16] that was also extended to six and seven external gluons [17,18]. During the final stages of this work, benchmark results for planar five-gluon amplitudes with generic helicity assignments were presented [19]. The amplitudes were integrated with a combination of numeric and analytic techniques, after partial reduction using unitarity cuts.

In this paper, we apply the numerical unitarity approach [9,20-22], which was recently extended to two-loops [23-25], to compute the full set of planar five-gluon two-loop amplitudes. This computation marks the first time that a full set of two-loop five-scale amplitudes is reduced to a linear combination of integral coefficients multiplied by corresponding master integrals. The set of master integrals is minimal, as all possible integral relations have been imposed. The potential of this approach has recently been demonstrated in the context of planar fourgluon two-loop amplitudes [25], and in order to handle the 
complexity of the five-point amplitudes, we refine it in the present work in two ways.

First, we use an improved approach to obtain analytic expressions for integration-by-parts (IBP) identities which are important in our construction of the amplitude. The unitarity method [5-8] matches unitarity cuts to an ansatz of the loop amplitudes written in terms of master integrals. When considered at the integrand level, one-loop unitarity approaches $[9,21]$ relate cuts to an ansatz for the amplitude's integrand written in terms of surface terms (spurious numerators that integrate to zero) and master integrands (the integrands corresponding to the master integrals). The numerical unitarity method at the two-loop level [23-25] is a natural generalization of the one-loop integrand level approach: the unitarity cuts are matched to a complete parametrization of the integrand in terms of specially tailored IBP relations [26-28] (the surface terms) and master integrands. As such, the method directly achieves a reduction to master integrals at the integrand level. Compared to more canonical approaches, the challenging inversion of the IBP systems required to obtain reduction tables for tensor integrals [27] is avoided. While we leave a more detailed discussion of the way we generate the required IBP relations to future work [29], we describe the central formal improvements for their derivation.

Second, we adapt numerical unitarity to exact numerical computations based on the finite field approach [30] and its application to unitarity cuts [31]. The finite field arithmetics allow us to compute exact values for the integral coefficients at rational phase-space points. Combined with the analytic expressions for two-loop master integrals with five massless external legs [15], we obtain stable results whose precision only depends on the number of digits to which the multiple polylogarithms appearing in the master integrals are evaluated. The extension to finite fields will be crucial for a future use of functional reconstruction techniques to determine analytic forms of the amplitudes from the exact numerical results.

The article is organized as follows. In Sec. II, we summarize the numerical unitarity method, as well as the two refinements already highlighted. In Sec. III, we give explicit details of our implementation. In Sec. IV, we present our results for the four independent leading-color helicity amplitudes required for all five-gluon scattering processes at a given phase-space point. In Sec. V, we give our conclusions and outlook. Finally, in Appendix, we discuss the universal infrared structure of the amplitudes which we use to validate our results.

\section{NUMERICAL UNITARITY APPROACH}

The main goal of this paper is the calculation of two-loop corrections to five-gluon scattering amplitudes in the leading-color approximation for any helicity configuration in pure Yang-Mills theory. We write the perturbative expansion of a bare five-gluon helicity amplitude as

$$
\begin{aligned}
& \left.\mathcal{A}\left(\left\{p_{i}, h_{i}\right\}_{i=1, \ldots, 5}\right)\right|_{\text {leading color }} \\
& =\sum_{\sigma \in S_{5} / Z_{5}} \operatorname{Tr}\left(T^{a_{\sigma(1)}} T^{a_{\sigma(2)}} T^{a_{\sigma(3)}} T^{a_{\sigma(4)}} T^{a_{\sigma(5)}}\right) \\
& \quad \times g_{0}^{3}\left(\mathcal{A}^{(0)}+\frac{\alpha_{0} N_{C}}{4 \pi} \mathcal{A}^{(1)}+\left(\frac{\alpha_{0} N_{C}}{4 \pi}\right)^{2} \mathcal{A}^{(2)}+\mathcal{O}\left(\alpha_{0}^{3}\right)\right),
\end{aligned}
$$

where $\alpha_{0}=g_{0}^{2} /(4 \pi)$ is the bare QCD coupling. The set $S_{5} / Z_{5}$ denotes all noncyclic permutations of five indices, and we make it explicit that the color structures beyond tree-level give only a factor of $N_{C}$ at each order in the expansion in the leading-color approximation. The planar amplitudes $\mathcal{A}^{(j)}$ are functions of the momenta $p_{\sigma(i)}$, helicities $h_{\sigma(i)}$ and color indices $a_{\sigma(i)}$. In the leading-color approximation there is a single color structure and it is thus sufficient to specify a helicity assignment for the ordered set of legs,

$$
\mathcal{A}^{(i)}\left(1^{h_{1}}, 2^{h_{2}}, 3^{h_{3}}, 4^{h_{4}}, 5^{h_{5}}\right) .
$$

In this section, we describe our approach to the calculation of the $\mathcal{A}^{(i)}$. Although in this paper we focus on the leadingcolor contributions, the approach we use is completely generic and applicable beyond this approximation.

\section{A. Overview}

We apply a variant of the unitarity method [5-8] suitable for automated numerical computations of multiloop amplitudes [23-25]. Our approach starts from the standard decomposition of the amplitude in terms of master integrals,

$$
\mathcal{A}^{(k)}=\sum_{\Gamma \in \Delta} \sum_{i \in M_{\Gamma}} c_{\Gamma, i} \mathcal{I}_{\Gamma, i},
$$

where the index $\Gamma$ labels the different propagator structures and $i$ denotes an additional degeneracy for cases where multiple master integrals with identical propagator structures appear, as can happen beyond one loop. The set $\Delta$ denotes a hierarchy of propagator structures, where descendants are obtained from parent diagrams through pinches. The set $\Delta$ is the collection of diagrams which specify the possible propagator structures of the amplitude, and only depends on the kinematics of the amplitude (i.e., on the momenta and masses of the particles involved). In Sec. III, we give an explicit example for a set of propagator structures $\Delta$ and the corresponding set of master integrals together with their multiplicity in the case of two-loop fivegluon leading-color amplitudes. The aim of the computation is to determine the coefficient functions $c_{\Gamma, i}$ which contain the process specific information.

Next, we promote the decomposition of the amplitude of Eq. (2.3) to the integrand level. The integrand, which we 
denote $\mathcal{A}\left(\ell_{l}\right)$ with $\ell_{l}$ representing the loop momenta $\ell_{1}, \ldots, \ell_{L}$ in a $L$-loop calculation, is decomposed as [23]

$$
\mathcal{A}^{(k)}\left(\ell_{l}\right)=\sum_{\Gamma \in \Delta} \sum_{i \in M_{\Gamma} \cup S_{\Gamma}} \frac{c_{\Gamma, i} m_{\Gamma, i}\left(\ell_{l}\right)}{\prod_{j \in P_{\Gamma}} \rho_{j}},
$$

where the $\rho_{j}$ are the inverse propagators and $P_{\Gamma}$ the set of propagators associated with the topology $\Gamma$. Compared with (2.3), we have extended the sum on $i$ to also go over the socalled surface terms in the set $S_{\Gamma}$, which vanish upon integration but are required to parametrize the integrand. We work in dimensional regularization with $D$ spacetime dimensions, and thus allow $D$-dependent surface terms. All in all, the decomposition (2.4) is rather universal, depending only on the set of diagrams $\Delta$ and the allowed power counting of the theory under consideration. As in the decomposition of Eq. (2.3), the spectrum-specific information is carried by the coefficients $c_{\Gamma, i}$. Obtaining a complete parametrization of the integrand of the amplitude is a nontrivial process, and in the next subsection, we explain how this was achieved. For now, it is sufficient to assume that such a decomposition exists.

The ansatz (2.4) holds as a function of the loop momenta $\ell_{l}$. Provided one can evaluate $\mathcal{A}^{(k)}\left(\ell_{l}\right)$ at specific values of $\ell_{l}$, it can be used to construct a system of equations for the coefficients $c_{\Gamma, i}$. In a generalized unitarity calculation, the coefficients $c_{\Gamma, i}$ are determined from a set of cut equations obtained by setting $\ell_{l}$ to specific on-shell configurations. Indeed, the leading terms in the various on-shell limits of the ansatz (2.4) factorize, yielding the cut equations

$$
\sum_{\text {states }} \prod_{i \in T_{\Gamma}} \mathcal{A}_{i}^{\text {tree }}\left(\ell_{l}^{\Gamma}\right)=\sum_{\substack{\Gamma^{\prime} \geq \Gamma^{\prime}, i \in M^{\prime} S_{\Gamma^{\prime}}}} \frac{c_{\Gamma^{\prime}, i} m_{\Gamma^{\prime}, i}\left(\ell_{l}^{\Gamma}\right)}{\prod_{j \in\left(P_{\Gamma^{\prime}} \backslash P_{\Gamma}\right)} \rho_{j}\left(\ell_{l}^{\Gamma}\right)} .
$$

Here, the set $T_{\Gamma}$ labels all tree amplitudes corresponding to the vertices in the diagram $\Gamma$, and the state sum runs over all internal states allowed by the theory. The $\ell_{l}^{\Gamma}$ correspond to a configuration of the loop momenta where the propagators in the set $P_{\Gamma}$ associated with the topology $\Gamma$ are on-shell. Although this limit probes all topologies $\Gamma^{\prime}$ for which $P_{\Gamma} \subseteq P_{\Gamma^{\prime}}$, through a top-down approach, i.e., starting from the topologies with the most propagators, we can make sure that the only unknowns in Eq. (2.5) are the coefficients $c_{\Gamma, i}$ belonging to $\Gamma$ itself. By sampling over enough values of $\ell_{l}^{\Gamma}$, we can build a system of equations big enough to determine all the coefficients. We note that beyond one loop there are topologies that correspond to subleading terms in the on-shell limit $\ell_{l}^{\Gamma}$, in which case no factorization of the integrand is known. This is a minor obstacle that can be easily overcome [24].

For the process we are concerned with in this paper, two-loop gluonic amplitudes in the leading-color approximation, the state sum in Eq. (2.5) goes over the $\left(D_{s}-2\right)$ gluon helicity states. Furthermore, as the surface terms are functions of the spacetime dimension parameter $D$, the coefficients $c_{\Gamma, i}$ obtained by solving the cut equations are functions of both $D_{s}$ and $D$. The value chosen for $D_{s}$ defines different regularization schemes. We have implemented both the 't Hooft-Veltman (HV) scheme [32], where $D_{s}=D=4-2 \epsilon$, and the four-dimensional helicity (FDH) scheme [33,34] where $D_{s}=4$.

\section{B. Efficient construction of unitarity-compatible IBP identities}

We now return to the discussion of how the decomposition in Eq. (2.4) is achieved. Here we present the generic framework of our method but leave more specific details for a future dedicated publication [29]. The surface terms are constructed from a complete set of so-called "unitarity-compatible" integration-by-parts (IBP) identities [23,35-37]. These relations are built such that they do not involve any new propagator structures besides those in $\Delta$, the full set of propagator structures we started with. As is the case in more canonical approaches to multiloop amplitude calculations, our approach requires a complete set of IBP identities for each of the propagator structures [25]. In the context of the ansatz in Eq. (2.4), completeness means that surface terms and master integrands span the full function space prescribed by the power counting of the theory. The system of equations in Eq. (2.5) is then invertible and by solving it we achieve a full reduction of the amplitude to master integrals.

In principle, the desired decomposition can be achieved by IBP reducing all allowed insertions of irreducible scalar products (ISPs) ${ }^{1}$ on a given propagator structure. The reduction identities may then be used as the set of surface terms in Eq. (2.4). In practice, the currently available reduction programs [38-44] struggle with the high tensor-rank appearing in two-loop five-gluon amplitudes as the size of the IBP system that needs to be solved becomes prohibitive. Instead, we follow an alternative path to obtain unitarity-compatible relations which has recently received much attention [23,25,35-37,45-48]. By only generating unitarity-compatible IBP relations from the start, we ensure that the size of the system remains manageable. In our approach, the solution of the IBP system is done by solving the cut equations (2.5), which allows to efficiently organize the calculation.

We start from the construction of the "transverse IBP identities" from Refs. [23,25], but further improve the construction of the remaining identities by solving syzygy module equations which we now briefly describe, leaving a more detailed presentation for a separate publication [29]. The way the syzygy module equations arise is closely related to the defining equation for the so-called IBPgenerating vectors $\left\{u_{k}^{\nu}\right\}$ [35],

\footnotetext{
${ }^{1}$ Precisely, ISPs are scalar products involving loop momenta that cannot be expressed solely in terms of inverse propagators.
} 


$$
u_{k}^{\nu} \frac{\partial}{\partial \ell_{k}^{\nu}} \rho_{j}=f_{j} \rho_{j}, \quad \forall j \in P_{\Gamma},
$$

where $1 \leq k \leq L$ is the loop momentum label, $\nu$ is the Lorentz index and there is no summation over $j$. We require $f_{j}$ to be polynomial in the dot products built from loop momenta and external momenta. The vectors $\left\{u_{k}^{\nu}\right\}$ are required to be polynomial vectors in the loop momenta. The above equation ensures that the IBP relation

$$
\int \prod_{l=1, L} d^{D} \ell_{l} \sum_{k} \frac{\partial}{\partial \ell_{k}^{\nu}}\left[\frac{u_{k}^{\nu}}{\prod_{j \in P_{\Gamma}} \rho_{j}}\right]=0
$$

contains no integrals with raised propagator powers and is therefore a suitable unitarity-compatible IBP relation. In the context of Eq. (2.4), the left-hand side of (2.7) gives a valid surface term.

Our method uses inverse propagator variables to trivialize the separation of terms that vanish on-shell from ISPs that survive on the cut, as in $[23,25]$. However, we further reduce the polynomial degree of the syzygy equations by simply rewriting the defining equation for IBP-generating vectors (2.6) in terms of the components of the loop and the external momenta as we shall see below. If starting from a formulation in terms of so-called Baikov polynomials [37], this method of solving Eq. (2.6) may be viewed as a variant of the intersection method [47]. We write down the following ansatz for the IBP-generating vector

$$
u_{k}^{\nu} \frac{\partial}{\partial \ell_{k}^{\nu}}=\left(u_{k a}^{\text {loop }} \ell_{a}^{\nu}+u_{k c}^{\text {ext }} p_{c}^{\nu}\right) \frac{\partial}{\partial \ell_{k}^{\nu}},
$$

where we have an implicit sum over the label $a$ for independent loop momenta and label $c$ for independent external momenta. Consistent with the fact that the original vectors $\left\{u_{k}^{\nu}\right\}$ are polynomial we require the vector components $u_{k a}^{\text {loop }}$ and $u_{k c}^{\text {ext }}$ to be a polynomial in the dot products built from loop momenta and external momenta. Since the latter can be expressed in terms of ISPs and inverse propagators, the variables $u_{k a}^{\text {loop }}, u_{k c}^{\text {ext }}$ and $f_{j}$ are polynomials in ISPs and inverse propagators. Equation (2.6) then becomes

$$
\left(u_{k a}^{\text {loop }} \ell_{a}^{\nu}+u_{k c}^{\text {ext }} p_{c}^{\nu}\right) \frac{\partial}{\partial \ell_{k}^{\nu}}\left(\begin{array}{c}
\rho_{j(1)} \\
\rho_{j(2)} \\
\vdots \\
\rho_{j(|\Gamma|)}
\end{array}\right)-\left(\begin{array}{c}
f_{j(1)} \rho_{j(1)} \\
f_{j(2)} \rho_{j(2)} \\
\vdots \\
f_{j(|\Gamma|)} \rho_{j(|\Gamma|)}
\end{array}\right)=0,
$$

where there is implicit summation over $a, c, k$, and $\nu$, and the inverse propagator labels $j(i)$ run over all propagators in the set $P_{\Gamma}$,

$$
\{j(1), j(2), \ldots, j(|\Gamma|)\} \equiv P_{\Gamma} .
$$

Note that both

$$
\ell_{a}^{\nu} \frac{\partial}{\partial \ell_{k}^{\nu}} \rho_{j} \quad \text { and } \quad p_{c}^{\nu} \frac{\partial}{\partial \ell_{k}^{\nu}} \rho_{j}
$$

evaluate to contractions of the propagator momenta with loop and external momenta, and can be expressed as matrices of linear polynomials in ISPs and inverse propagators. Put differently, these are polynomials of degree one in the dot products of internal and external momenta. This contrasts with what one would get in an approach based on the Baikov polynomial which has degree four in a two-loop calculation. Similarly, the second term in Eq. (2.9) can be expressed in matrix form with propagator variables on the diagonal. Consequently Eq. (2.9) has the form of a syzygy equation over a module for the unknown polynomials $u_{k a}^{\text {loop }}$, $u_{k c}^{\text {ext }}$ and $f_{j}$. The equations are defined over the freely generated polynomial ring given by ISPs and inverse propagators. Syzygy equations can be solved using algorithms in computational algebraic geometry implemented in e.g., the Singular computer algebra system [49]. We will give more details of how we solved the syzygy equations using SINGULAR in the next section.

Once a generating set of vector components $u_{k a}^{\text {loop }}$ and $u_{k c}^{\mathrm{ext}}$ has been obtained, a sufficient set of IBP relations is obtained by multiplying the generators with irreducible numerators. At this stage, we explicitly impose off-shell power-counting conditions (i.e., we consider both on-shell and propagator variables), sometimes after forming linear combinations of IBP relations. In a second step the independence of IBP identities has to be established. Given the manifest dependence on propagator variables and the vectors compatibility with unitarity cuts, it is natural to switch between on-shell and off-shell as well as numerical and analytic perspectives to simplify computational steps $[23,37]$. In particular, the validation of the linear independence of the off-shell relations $[23,25]$ is most easily performed by setting propagator variables to their on-shell values, as redundancy of the relations is then manifest. Finally, we note it is often convenient to count the number of master integrals on-shell $[23,25,37,45]$.

\section{Numerical unitarity over an arbitrary number field}

Numerical computations have better scaling properties than analytic ones for processes with a large number of scales. However, the stability and efficiency of analytic results for amplitudes often offer major advantages over numerical evaluations, specially when used in conjunction with Monte Carlo programs. The boundary between the two approaches is becoming blurred in the field of scattering amplitude calculations with the recent introduction of functional reconstruction techniques [25,30,31]. 
In this approach, numerical evaluations are used to completely reconstruct analytic expressions from numerical samples. While already successful in the reconstruction of two-loop amplitudes with two scales [25], it is clear that multiscale problems such as the five-gluon amplitudes are significantly more complicated. Not only must more efficient techniques be used for the functional reconstruction [31], but the loss of precision associated with floating-point arithmetics in multiscale computations poses a considerable difficulty for the reconstruction procedure. To address this issue, in this section, we reformulate numerical unitarity for gluonic amplitudes so that it only employs operations defined on a field (addition, subtraction, multiplication and division). In this way, we open the door to the use of exact arithmetics, such as those of rational numbers or finite fields. This eliminates any question of numerical stability, whilst still leveraging the speed of modern computer hardware.

The extension of numerical unitarity to employ only field operations is nontrivial. Indeed, operations such as taking square roots or more generally solving generic polynomial equations are not allowed, and these appear prominently in many formulations of generalized unitarity. Here, we build on the ideas proposed in Ref. [31] and use them in the context of multiloop numerical unitarity. More concretely, there are two components of a typical numerical unitarity formulation that must be re-examined: (1) the generation of a set of four-momenta satisfying momentum conservation and on-shell conditions for the external kinematics, and (2) the parametrization of the internal loop momenta, in particular for the solution of the quadratic on-shell equations and the construction of specific sets of surface terms. In this section, we will describe how these obstacles can indeed be overcome. Furthermore, this will be achieved without needing to introduce any notion of complex numbers. In the following we work over an arbitrary field which we denote by $\mathbb{F}$, and which for all practical purposes can be considered to be the rational numbers $\mathbb{Q}$.

\section{External kinematics}

The problem of generating a set of $\mathbb{F}$-valued external momenta which satisfy on-shell conditions as well as momentum conservation can be solved in a number of ways. In this work, we choose to parametrize the external kinematics based upon so-called "momentum twistors" [50] as proposed in Refs. [12,31], where the reader can find further details. In short, momentum twistors are particularly useful because they trivialize the on-shell and the momentum conservation conditions. By starting with an $\mathbb{F}$-valued parametrization in twistor space, one obtains $\mathbb{F}$-valued spinors associated to each momentum. If working with the usual Minkowski metric $\operatorname{diag}\{+1,-1,-1,-1\}$, the associated four-momenta and polarization vectors would involve explicit factors of $i$ and thus require an extension of $\mathbb{F}$. This can be avoided by using the metric $\operatorname{diag}\{+1,-1,+1,-1\}$, in which case the momenta and polarization vectors constructed from the $\mathbb{F}$-valued spinors are themselves $\mathbb{F}$-valued. ${ }^{2}$ Avoiding the introduction of complex values offers a considerable speed boost in a numerical implementation. We also note that a wisely chosen parametrization such as that of [31] means that the invariants and Gram-determinants are compact functions of the parameters. This simplifies the functional dependence of the amplitude on these parameters, making them particularly suitable for a future reconstruction of the analytic expressions.

\section{On-shell momenta}

As discussed around Eq. (2.5), in order to compute integral coefficients in a unitarity approach we need to generate loop momenta which satisfy a set of conditions which set some propagators on-shell. For a two-loop calculation, this set of topology specific, kinematicallydependent quadratic conditions define an algebraic variety in the six-dimensional space in which we embed the loop momenta. A direct approach for finding a rational parametrization of this variety, i.e., a parametrization in terms of a set of $\mathbb{F}$-valued parameters that only uses the operations that are defined on a field, is nontrivial. Instead we take inspiration from the fact that the integrand is a rational function of irreducible scalar products (ISPs), and the $\mu_{i j}$ variables which we shall define shortly. We will see that in a set of adapted coordinates it is trivial to generate loop momenta such that the ISPs and $\mu_{i j}$ are $\mathbb{F}$-valued. Therefore, the integrand evaluated on such a loop-momentum configuration will also be $\mathbb{F}$-valued. We then represent the loop momenta in a phase-space dependent way, circumventing the rational parametrization required when using the standard six-dimensional representation. We now give more details about this procedure.

We begin by parametrizing the loop momenta $\ell_{l}(l=1$, 2, 3 see Fig. 1) as $[23,25]$

$$
\begin{gathered}
\ell_{l}=\sum_{j \in B_{l}^{p}} v_{l}^{j} r^{l j}+\sum_{j \in B_{l}^{t}} v_{l}^{j} \alpha^{l j}+\sum_{i \in B^{c t}} \frac{n^{i}}{\left(n^{i}\right)^{2}} \alpha^{l i}+\sum_{i \in B^{\epsilon}} n^{i} \mu_{l}^{i}, \\
r^{l j}=-\frac{1}{2}\left(\rho_{l j}-\left(q_{l j}\right)^{2}-\rho_{l(j-1)}+\left(q_{l(j-1)}\right)^{2}\right), \\
\mu_{l l}=\rho_{l 0}-\sum_{\nu=0}^{3} \ell_{l}^{\nu} \ell_{l \nu},
\end{gathered}
$$

\footnotetext{
${ }^{2}$ In the standard formulas for gluonic polarization vectors, there is an explicit factor of $\sqrt{2}$ :

$$
\epsilon_{+}^{\mu}(p, \eta)=\frac{\left\langle\eta\left|\sigma^{\mu}\right| p\right]}{\sqrt{2}\langle\eta p\rangle}, \quad \epsilon_{-}^{\mu}(p, \eta)=\frac{\left\langle p\left|\sigma^{\mu}\right| \eta\right]}{\sqrt{2}[p \eta]} .
$$

This is not an issue as it can be restored as a global factor after computation.
} 


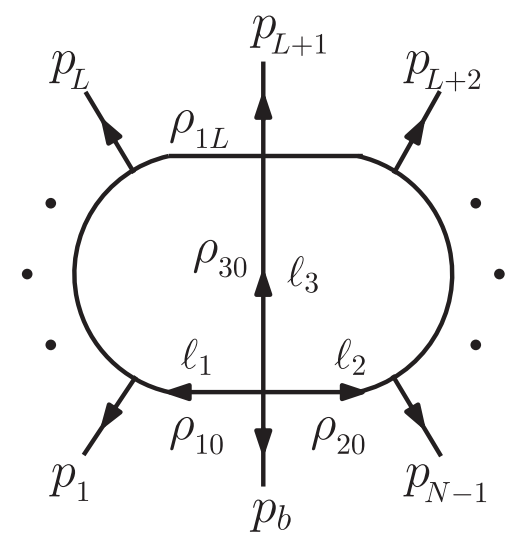

FIG. 1. Displayed are the conventions for assigning propagators in a two-loop diagram.

which are related by momentum conservation, $\ell_{1}+\ell_{2}+$ $\ell_{3}+p_{b}=0$. We use a total of 11 variables consisting of the ISP variables $\alpha^{l i}(l=1,2)$ and all propagator variables $\rho_{l j}(l=1,2,3)$ as independent variables. The remaining dependent ISP variables $\alpha^{l i}(l=3)$ are obtained from momentum conservation and the $\mu_{i j}$ variables are obtained from the linear relations in Eq. (2.15). The redundant variables $\mu_{l}^{i}$ do not explicitly appear in our computations as described in Sec. III, and we do not require them. The vectors $q_{l j}$ are linear combinations of the external momenta $p_{i}$. The loop momenta are parametrized in terms of socalled adapted coordinates: the inverse propagator variables $\rho_{l i}$ and the auxiliary variables $\alpha^{l i}$ and $\mu_{l}^{i}$. The variables $\mu_{l}^{i}$ are dependent and fixed by (2.15). The vectors $n^{i}$ form an orthogonal basis transverse to the scattering plane, i.e. $n^{i} \cdot p_{j}=0$. Labels in $B^{\epsilon}$ refer to directions beyond four dimensions and labels in $B^{c t}$ denote directions within four dimensions transverse to the scattering plane defined by the momenta $p_{i}$. For each strand $l$ of the diagram we use a distinct basis of the scattering plane, spanned by the vectors $v_{l}^{i}$,

$$
v_{l}^{i}=\left(G_{l}\right)^{i j} p_{j}, \quad \text { with } \quad i, j \in B_{l}^{p} \cup B_{l}^{t},
$$

where $\left(G_{l}\right)^{i j}$ is the inverse of the Gram matrix,

$$
\left(G_{l}\right)_{i j}=p_{i} \cdot p_{j} \quad \text { with } \quad i, j \in B_{l}^{p} \cup B_{l}^{t} .
$$

The index set $B_{l}^{p}$ labels the external momenta which leave the strand $l$. These momenta are completed with other independent external momenta $p_{i}$, with $i \in B_{l}^{t}$, so as to span the whole scattering plane. This parametrization follows the conventions of Ref. [25], with the caveat that the vectors spanning $B^{c t}$ are no longer normalized.

The inverse coordinate transformation is often useful and is given by

$$
\alpha^{l i}=p_{i} \cdot \ell_{l}, \quad i \in B_{l}^{t},
$$

$$
\begin{gathered}
\alpha^{l i}=n^{i} \cdot \ell_{l}, \quad i \in B^{c t}, \\
\rho_{l i}=\left(\ell_{l}-q_{l i}\right)^{2}
\end{gathered}
$$

The on-shell variety is then defined by setting the propagator variables $\rho_{l i}$ to zero. In $D$ dimensions, the variables $\alpha^{l i}$ form an independent complete set of coordinates on the variety, corresponding to polynomials in momentum variables. They are the irreducible scalar products we already mentioned previously.

By considering the $\alpha^{l i}$ as a set of independent coordinates and taking them as $\mathbb{F}$-valued, the constraint equations (2.15) imply that the $\mu_{i j}$ are also $\mathbb{F}$-valued. We must now construct an explicit representation of the loop momenta. This is required, for instance, for the calculation of the tree amplitudes in Eq. (2.5). In the fourdimensional slice, this is trivial to achieve with a standard cartesian basis. However, if we were to also do this for the $(D-4)$-dimensional space we would generically be required to take square roots.

We now present our solution to this problem in the context of a two-loop calculation, but it trivially generalizes to any loop order. The main idea is to employ a different basis of the $(D-4)$-dimensional space for each on-shell phase-space point. This is achieved by picking basis vectors which are linear combinations of the $(D-4)$ dimensional components of the loop momenta. Given the two loop momenta,

$$
\ell_{1}=\left(\ell_{1,[4 d]}, \vec{\mu}_{1}\right), \quad \ell_{2}=\left(\ell_{2,[4 d]}, \vec{\mu}_{2}\right),
$$

and their $(D-4)$-dimensional parts $\vec{\mu}_{1}$ and $\vec{\mu}_{2}$, we construct the orthogonal basis vectors ${ }^{3}$

$\tilde{n}^{1}=\left(0_{[4 d]}, \vec{\mu}_{1}\right), \quad \tilde{n}^{2}=\left(0_{[4 d]}, \vec{\mu}_{2}-\frac{\mu_{12}}{\mu_{11}} \vec{\mu}_{1}\right)$,

with $\mu_{i j}=-\vec{\mu}_{i} \cdot \vec{\mu}_{j}$, here using the Euclidean scalar product. We stress that the basis vectors $\tilde{n}^{1}$ and $\tilde{n}^{2}$ used to represent the $(D-4)$-dimensional space are no longer of unit norm. For each on-shell point, which corresponds to a different value of the $\mu_{i j}$, this affects how we calculate the scalar product between two vectors $w_{a}$ and $w_{b}$. Explicitly,

$$
\begin{gathered}
w_{a} \cdot w_{b}=w_{a,[4 d]} \cdot w_{b,[4 d]}+w_{a}^{5} w_{b}^{5}\left(\mu_{11}\right) \\
+w_{a}^{6} w_{b}^{6}\left(\mu_{22}-\frac{\mu_{12}^{2}}{\mu_{11}}\right) .
\end{gathered}
$$

\footnotetext{
${ }^{3}$ Also at higher loops, one can construct orthogonal combinations through a modified Gram-Schmidt procedure.
} 
Such a scalar product then allows $\mathbb{F}$-valued representations of loop momenta which satisfy the on-shell conditions for an arbitrary topology.

This momentum representation is almost all that is required to use a Berends-Giele recursion [51] to calculate products of tree amplitudes. The remaining difficulty is in performing the sum over helicities at each cut line, as this requires explicit representations of the $D_{s}$-dimensional polarization vectors. To remedy this, we choose to avoid constructing the polarization states by trading the helicity sum for the insertion of a light-cone projection operator in the $D_{s}$-dimensional space of the polarization vectors (see e.g., [52]),

$$
P_{l}^{\mu \nu}=-g^{\mu \nu}+\frac{\ell_{l}^{\mu} \eta^{\nu}+\eta^{\mu} \ell_{l}^{\nu}}{\eta \cdot \ell_{l}}
$$

where $\eta$ is an arbitrary $\mathbb{F}$-valued light-like reference vector satisfying $\eta \cdot \ell_{1} \neq 0$ and $\eta \cdot \tilde{n}=0$. Note that in all but one cut line per loop, the Ward identity allows us to drop the second term of Eq. (2.24). For the remaining cut line of each loop, we reexpress the projector as a sum of a direct product of vectors over $\mathbb{F}$.

We note that as the irreducible scalar products of Eq. (2.19) are expressed in terms of a set of non-normalized vectors, this affects the representation of so-called "traceless completion" surface terms [25], i.e., surface terms associated with variables in $B^{c t} \cup B^{\epsilon}$. This normalization now explicitly appears in the parametrization of the loop momenta, see Eq. (2.13). For example, consider a traceless completion surface term associated to the transverse vector $n^{i}, i \in B^{c t}$,

$$
\frac{\alpha_{i}^{2}}{n_{i}^{2}}-\frac{\mu_{11}}{D-4}
$$

Only by including the factor of $n_{i}^{2}$ is the numerator insertion (2.25) a surface term.

We finish this section by noting that this procedure applies both for planar and nonplanar cases, and is easily generalized to higher (and lower) loops. In summary, using the steps described in Secs. II C 1 and II C 2, all contributions are manifestly $\mathbb{F}$-valued, and as an added benefit we never needed to introduce complex numbers.

\section{IMPLEMENTATION FOR PLANAR FIVE-GLUON AMPLITUDES}

We have implemented the techniques described in Sec. II in a $\mathrm{C}++$ code. In this section, we first discuss the implementation of the cut equations. Then we describe how these are solved in practice to compute the master integral coefficients and, finally, how we obtain the amplitude at a given kinematic point.

\section{A. Construction of cut equations}

We first construct the full set of propagator structures that appear in the problem (see Fig. 2). This is achieved by generating all cut diagrams for the full color process using QGRAF [53] and then color decomposing them according to [54] in MathEMATiCA. By taking the leading-color limit of this decomposition and extracting the coefficient of a given trace, we build the set of propagator structures relevant for the color ordered amplitude, which we then organize hierarchically. This is then passed on to a $\mathrm{C}++$ code.

The next step in constructing the cut equations is the parametrization of the integrand of the amplitude in terms of master integrands and surface terms. In order to solve the syzygy equations described in Sec. II B for each topology in Fig. 2, we use the package Singular. For these computations we find that the "slimgb" algorithm is much faster than the classical Buchberger algorithm. Details on the algorithm can be found in Ref. [55]. In a nutshell, "slimgb" uses weighted lengths of polynomials to make choices in key steps of the computation, in order to keep the size of intermediate results small. For complicated topologies we further speed up the computation by imposing a degree bound in SINGULAR, which restricts the maximum allowed polynomial degree in internal computations. Another significant speed-up is achieved by promoting parameters of the polynomial ring, which are external kinematic invariants, to variables of the polynomial ring, so that the solutions are restricted to have polynomial (instead of rational) dependence on these kinematic invariants. In practice, and despite the fact that the degree bound becomes a more stringent restriction, this does not prevent us from finding the solutions that lead to a complete set of IBP relations. For one of the most complicated topologies involved in our calculation, namely the pentabox shown on the top left of Fig. 2, our method succeeds in finding fully off-shell IBP-generating vectors, with analytic dependence on all external kinematic invariants, in under a second. The computation used only one CPU core on a modern laptop computer. Once the IBP-generating vectors have been obtained, the IBP system is constructed in a second step by multiplying the generating vectors with monomials in the ISP variables and computing the IBP relations. The linear dependence and completeness of the IBP system is determined on-shell and on a numerical kinematic point. All surface terms have been validated with Fire [39,40] on a fixed numerical kinematic point.

We note that a fast evaluation of the surface terms is required for an efficient implementation. To this end we express the surface terms as IBP-generating vector components multiplied by derivatives of the irreducible scalar products, as well as the total divergences of the vectors multiplied by irreducible scalar products. Furthermore, to improve both evaluation time and compilation time of the IBP vector components, we found it useful to employ the facilities provided by FORM [56] for the simplification of 


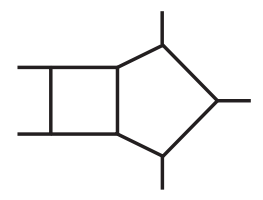<smiles>CC1C(C)C2C(C)C2C1C</smiles><smiles>CC1CC2CCC1C(C)C(C)C2C</smiles>
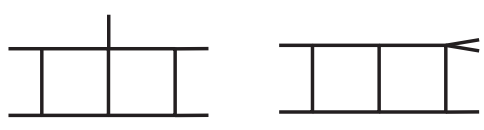<smiles>CC1CCC(C)C(C)C1C</smiles><smiles>CC1CCC(C)C(C)C1C</smiles><smiles>CC1CC2(C)CC(C)C(C2)C1C</smiles><smiles>CC1C(C)C2C(C)C1C2C</smiles><smiles>CC1C(C)C2C(C)C1C2C</smiles><smiles>CC1C(C)C2C(C)C1C2C</smiles><smiles>CC1C(C)C2C(C)C1C2C</smiles><smiles></smiles><smiles>CC1C2CCC1C(C1C3CC(C)(C(C)C3C)C1C)C2C</smiles><smiles>CC1CCC(C2CCC(C)C2C)C1C</smiles><smiles>CC1CCC(C)C1C</smiles><smiles>CC1CCCC1C</smiles><smiles>CC1CCCC1C</smiles><smiles>CC1CC2(C)CCC1C2C</smiles><smiles>C=C1C(C)CCC1C</smiles><smiles>CC1C(C)C2(C)C(C)C12C</smiles><smiles>CC1C2CC1C2(C)C</smiles><smiles>CC1C2C(C)C1(C)C2C</smiles>
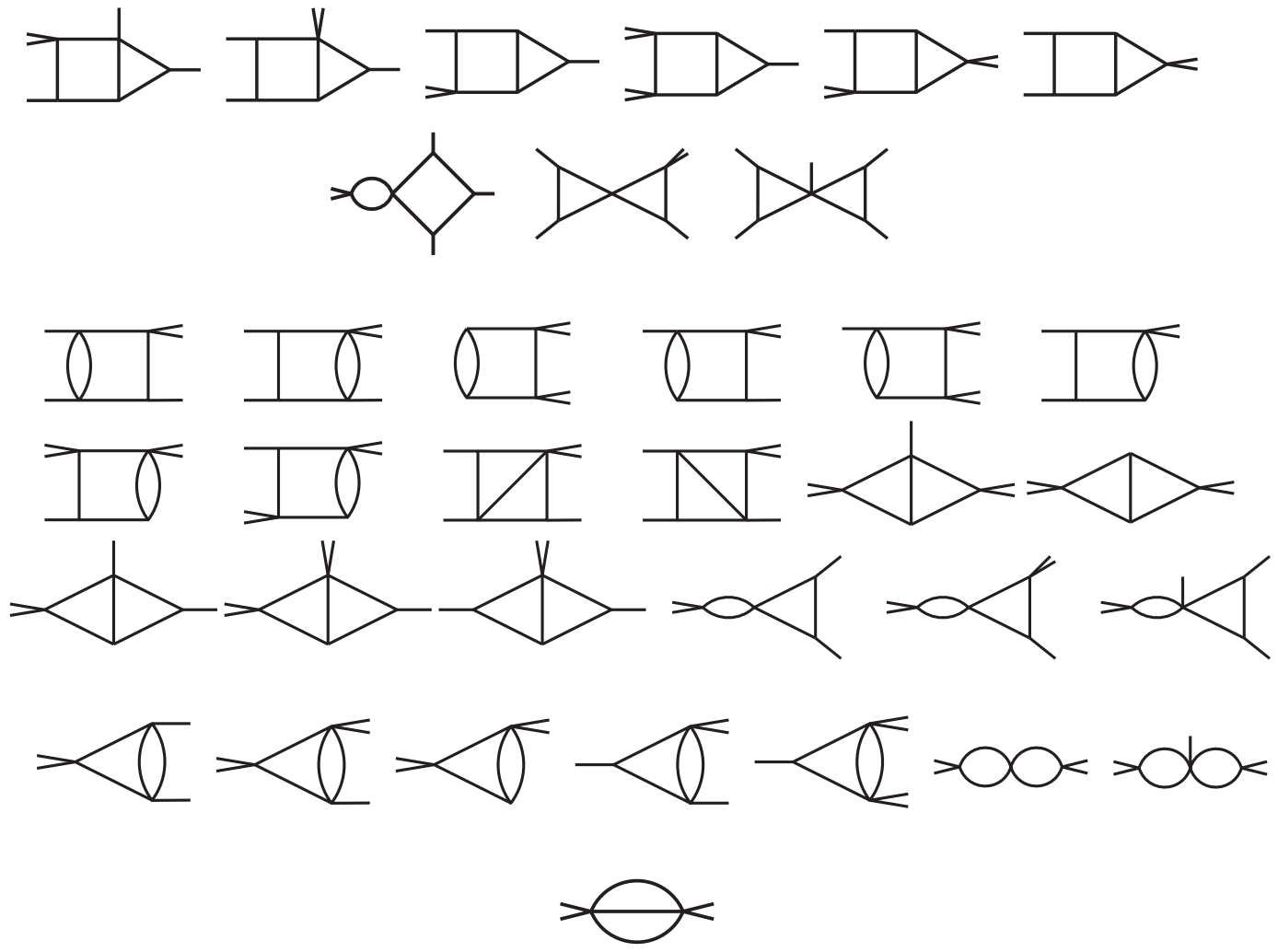

FIG. 2. Hierarchy of propagator structures for two-loop five-point gluon scattering as defined in Sec. II A. Only topologically inequivalent structures are shown. All lines are massless, with massive external legs being denoted by two massless external lines entering a vertex.

multivariate polynomials [57,58]. More precisely, FORM's \#optimize command implements a multivariate generalization of the so-called Horner's method to bring polynomials into a form that is computationally more efficient.
Given the parametrization of the integrand with surface terms, any linearly independent tensor insertion can be used as a master integral. As such, it is trivial for us to change basis of master integrals, by filling the master space of each 
topology with any set of integrals independent under the IBP relations. This freedom was especially useful during testing, where we could for instance make use of finite integrals to control the $\epsilon$ structure when these could be easily constructed (e.g., some five-point master integrals can be made finite by simply shifting them to six dimensions).

In order to numerically calculate the appropriate products of tree amplitudes necessary to reconstruct the integrand in our algorithm, we implement a Berends-Giele recursion [51] tailored to directly compute multiloop cuts using $D$-dimensional momenta and states living in $D_{s}$ dimensions. This is both a high-performance and flexible choice, as changing the field content requires only implementing new Feynman rules. The setup is particularly useful for our numerical computations in dimensional regularization, as it is straightforward to evaluate the products of tree amplitudes at different values of $D_{s}$ in order to reconstruct the functional dependence on the parameter, in a way that automatically recognizes if a given two-loop cut has a linear or quadratic dependence on $D_{s}$. Caching for multiple $D_{s}$ values is built-in. External momenta are taken to live in four dimensions, as do the associated gluonic polarization states. The $D$-dimensional loop momenta are represented in six dimensions, as described in detail in Sec. II C.

\section{B. Amplitude evaluation}

Having constructed the cut equations (2.5) as described in the previous section, we now solve them for the integral coefficients at fixed values of the kinematics. Both Eq. (2.5) and its hierarchically subtracted analogue describe linear systems in the ansatz coefficients. For fixed values of $D$ and $D_{s}$, we evaluate the equations numerically over enough randomly chosen on-shell momenta configurations to form a linear system that constrains the coefficients. We then solve this system for the coefficients using standard PLU factorization and back substitution.

In order to reconstruct the $D$ and $D_{s}$ dependence of the coefficients, we first sample $D_{s}$ over three distinct values [25] in a generalization of [21]. Indeed, a two-loop amplitude is a quadratic polynomial in $D_{s}$ which can be fully determined from its evaluation at three different values of $D_{s}$, thus allowing us to easily implement both the FDH and $\mathrm{HV}$ flavors of dimensional regularization. As $D_{s}$ is restricted to be greater than or equal to the embedding

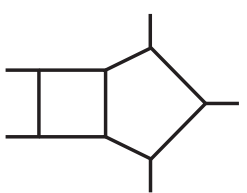

3 masters
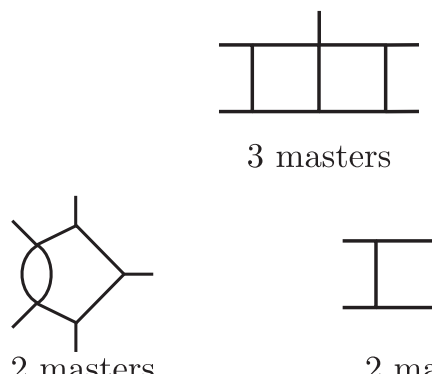

2 masters

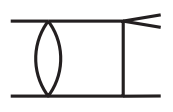

1 master

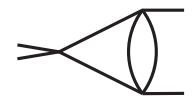

1 master
3 masters

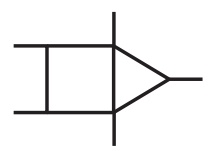

2 masters

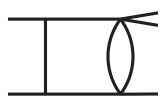

1 master

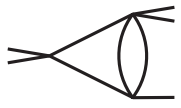

1 master

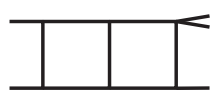

2 masters

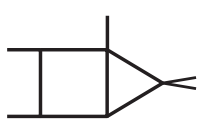

1 master

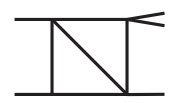

2 masters

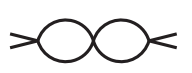

1 master

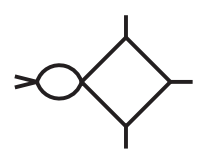

1 master

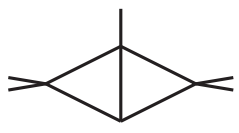

1 master

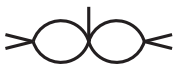

1 master

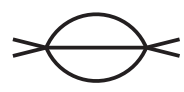

1 master

FIG. 3. Propagator structures with master integrals. 
TABLE I. Numeric results truncated to 10 significant figures for the two-loop all-plus and single-minus helicity amplitudes, normalized to the finite one-loop amplitudes truncated to leading order in $\epsilon$, at the kinematic point of Eq. (4.1). The normalization is fixed by Eq. (2.1).

\begin{tabular}{lcrr}
\hline \hline $\mathcal{A}^{(2)} /\left(\mathcal{A}^{(1)}(\epsilon=0)\right)$ & $\epsilon^{-2}$ & $\epsilon^{-1}$ & $\epsilon^{0}$ \\
\hline$\left(1^{+}, 2^{+}, 3^{+}, 4^{+}, 5^{+}\right)$ & -5.000000000 & -3.8931790255 & 5.9810885816 \\
$\left(1^{-}, 2^{+}, 3^{+}, 4^{+}, 5^{+}\right)$ & -5.000000000 & -16.322002103 & -10.383813287 \\
\hline \hline
\end{tabular}

space of the loop momenta (6 for a two-loop calculation) we pick $D_{s}=6,7,8$. The values of $D$ are chosen randomly. The $D$-dependence is obtained by repeating the computation for sufficient (a priori unknown) values of $D$ from which the rational dependence is reconstructed [30,31] using Thiele's formula [31,59]. After evaluation at a single phase-space point, the exact denominator as well as rank of the numerator function can be stored, allowing the use of simple polynomial inversion techniques and less sampling.

In practice, implementing our approach over the rational numbers $\mathbb{Q}$ will result in a slow calculation. However, the final master integral coefficients are strongly constrained by physical properties and it is expected that their resulting form will be compact. We thus follow the approach outlined in [30,31], using finite fields $\mathbb{F}_{p}$. We implement the algorithm over the finite fields provided by Givaro [60]. We use various cardinalities of order $2^{30}$, implementing Barrett reduction $[61,62]$ in order to improve the speed of finite field multiplication. For a given kinematic point in $\mathbb{Q}$, we perform the computation in a sufficient number of finite fields to apply a rational reconstruction algorithm, also provided by Givaro.

In order to obtain an $\epsilon$ expansion for the amplitudes, we combine the coefficients with $\epsilon$ expansions of the appropriate master integrals. We list the topologies with master integrals in Fig. 3. For the five-point master integrals we choose the basis of [15] in order to make use of the publicly available implementation distributed with the paper. For lower point integrals, we implemented the analytic expressions provided in [63]. In the case of factorizable topologies we choose the scalar integral as a master and calculate them independently. In order to evaluate the necessary multiple polylogarithms we use the implementation found in GiNaC [64], which can be tuned to the desired precision. All integrals have been independently numerically validated with Fiesta 4 [65]. We note that the choice [63] of an integral with a squared central propagator for the second slashed-box master is important in order to avoid spurious weight-five contributions in intermediate stages.

\section{RESULTS FOR HELICITY AMPLITUDES}

As an illustration of the implementation of our approach, we present numerical results for the four independent helicity configurations. We evaluate them at the Euclidean phase-space point ${ }^{4}$

$$
\begin{array}{ll}
p_{1}=\left(\frac{1}{2}, \frac{1}{16}, \frac{i}{16}, \frac{1}{2}\right), & p_{2}=\left(-\frac{1}{2}, 0,0, \frac{1}{2}\right) \\
p_{3}=\left(\frac{9}{2},-\frac{9}{2}, \frac{7 i}{2}, \frac{7}{2}\right), & p_{4}=\left(-\frac{23}{4}, \frac{61}{16},-\frac{131 i}{16},-\frac{37}{4}\right) \\
p_{5}=\left(\frac{5}{4}, \frac{5}{8}, \frac{37 i}{8}, \frac{19}{4}\right),
\end{array}
$$

which corresponds to the set of invariants (we write $\left.s_{i j}=\left(p_{i}+p_{j}\right)^{2}\right)$

$s_{12}=-1, \quad s_{23}=-8, \quad s_{34}=-10$,

$s_{45}=-7, \quad s_{51}=-3$,

and set the dimensional regularization scale $\mu$ to 1 . The normalization of the results we present is fixed by the expansion in Eq. (2.1), and they are all given in the HV scheme, $D_{s}=D=4-2 \epsilon$. Since our coefficients are exact rational numbers and we have analytic expressions for the master integrals $[15,63]$, the precision of the results we present is only limited by the number of digits we ask from $\mathrm{GiNaC}$ [64] when evaluating the master integrals, which can be arbitrarily increased.

The results for the all-plus and single-minus helicity amplitudes, $\mathcal{A}^{(2)}\left(1^{+}, 2^{+}, 3^{+}, 4^{+}, 5^{+}\right)$and $\mathcal{A}^{(2)}\left(1^{-}, 2^{+}, 3^{+}\right.$, $\left.4^{+}, 5^{+}\right)$, are given in Table I. Both helicity configurations vanish at tree-level, which implies they are finite at one-loop and start at order $\epsilon^{-2}$ at two-loops. We present our two-loop results normalized to $\mathcal{A}^{(1)}(\epsilon=0)$, the finite one-loop amplitude truncated to leading order in $\epsilon$. The results for the two independent MHV amplitudes, the split $\mathcal{A}^{(2)}\left(1^{-}, 2^{-}, 3^{+}, 4^{+}, 5^{+}\right)$and the alternating $\mathcal{A}^{(2)}\left(1^{-}, 2^{+}\right.$, $\left.3^{-}, 4^{+}, 5^{+}\right)$helicity configurations, are given in Table II. In this case, we normalize the results to $\mathcal{A}^{(0)}$, the corresponding tree-level amplitude. As expected, the first two leading poles are helicity independent.

\footnotetext{
${ }^{4}$ The reason for the explicit factor of $i$ in the third component of the four-vectors is that we write them here for the standard $\operatorname{diag}(+,-,-,-)$ metric. In our implementation we use the metric $\operatorname{diag}(+,-,+,-)$, see Sec. II C 1, in which case all the components are real.
} 
TABLE II. Numeric results truncated to 10 significant figures for the two-loop split and alternating MHV amplitudes, normalized to the tree level, at the kinematic point of Eq. (4.1). The normalization is fixed by Eq. (2.1).

\begin{tabular}{lccccc}
\hline \hline $\mathcal{A}^{(2)} / \mathcal{A}^{(0)}$ & $\epsilon^{-4}$ & $\epsilon^{-3}$ & $\epsilon^{-2}$ & $\epsilon^{-1}$ & $\epsilon^{0}$ \\
\hline$\left(1^{-}, 2^{-}, 3^{+}, 4^{+}, 5^{+}\right)$ & 12.5000000 & 25.46246919 & -1152.843107 & -4072.938337 & -3637.249566 \\
$\left(1^{-}, 2^{+}, 3^{-}, 4^{+}, 5^{+}\right)$ & 12.5000000 & 25.46246919 & -6.121629624 & -90.22184214 & -115.7836685 \\
\hline \hline
\end{tabular}

TABLE III. Numeric results for the one-loop all-plus and single-minus amplitudes normalized to their $\epsilon^{0}$ coefficient at the kinematic point of Eq. (4.1) used in checks of the universal pole structure.

\begin{tabular}{lc}
\hline \hline $\mathcal{A}^{(1)} /\left(\mathcal{A}^{(1)}(\epsilon=0)\right)$ & $\epsilon$ \\
\hline$\left(1^{+}, 2^{+}, 3^{+}, 4^{+}, 5^{+}\right)$ & 0.310137038 \\
$\left(1^{-}, 2^{+}, 3^{+}, 4^{+}, 5^{+}\right)$ & 2.795901653 \\
\hline \hline
\end{tabular}

To validate our results, we first reproduce the universal ultraviolet/infrared pole structure of the amplitudes [66], which we summarize in Appendix in the context of twoloop five-gluon amplitudes in the leading-color approximation. Computing the prediction for the pole structure requires the five-point one-loop amplitudes up to order $\epsilon$ for all four independent helicity configurations. For this, we used our own implementation of numerical unitarity at one loop, which we checked up to order $\epsilon^{0}$ against results from BLACKHAT [22]. The one-loop coefficients are computed in high precision and we use analytic expressions for all oneloop master integrals to obtain the 10 significant digits given in Tables III and IV of Appendix. Using our code, we confirm the published results for the all-plus helicity amplitudes $[3,14,16]$. We also validate the results of [19] which appeared during the final stages of the preparation of this article.

The computation of the master coefficients for each of the helicity amplitudes presented in Tables I and II involves the sampling of a large amount of trees over on-shell phase spaces. This is performed over ten values of $D$ and three values of $D_{s}$ to fully reconstruct the dependence on the dimensional regulator. For the most complex amplitude considered and with our current implementation, the determination of the full set of master coefficients in a single finite field takes around two and a half minutes in a single-threaded calculation employing an $i 7$ Intel processor. Then, reconstructing the exact rational coefficients used in obtaining the final results presented required the evaluation over seven finite fields.
Finally, we note that although we only present results for the four independent helicity configurations, we have also verified the pole structure for the other helicity configurations obtained by parity conjugation or permutation of external legs. Since our calculation is based on a numerical setup, these amount to independent calculations that give us an internal consistency check of our implementation.

\section{CONCLUSION}

The main result of this paper is a numerical implementation of two-loop five-gluon amplitudes in the leading-color approximation, for any helicity configuration. The calculation is done in the multiloop numerical unitarity approach, which we extended for use with finite-field arithmetics. Using a new approach to the generation of IBP relations, we obtain a complete decomposition of the amplitude in terms of master integrals. Because of the extension to finite-field arithmetics, the corresponding coefficients computed from unitarity cuts are exact. To illustrate our calculation, we presented the results at a given kinematic point, for which we used the available master integrals $[15,63]$. Our implementation was validated by checking the universal pole structure [66], and confirms all results available in the literature $[3,14,16,19]$.

The high polynomial degree and the many scales associated with multiloop computations with several external particles make it challenging to set up accurate numerical approaches. While analytic results are stable, the complexity of intermediate computational steps often makes them difficult to obtain for challenging amplitudes of that kind. Here, we chose an alternative approach which removes the strict separation of numerical and analytic perspectives and keeps the best features of each. We employed exact arithmetics as previously explored in the context of generalized unitarity in Ref. [31]. Our approach is universal and is obtained after refinements of the method introduced in $[24,25]$. The exact approach is valuable for a number of reasons: the validation of results is simplified because the results are exact, which allows us to evaluate the amplitudes in singular limits, and, in addition, it paves the way for the functional reconstruction of their analytic

TABLE IV. Numeric results for the one-loop MHV amplitudes normalized to the tree amplitude at the kinematic point of Eq. (4.1) used in checks of the universal pole structure.

\begin{tabular}{lcccc}
\hline \hline $\mathcal{A}^{(1)} / \mathcal{A}^{(0)}$ & $\epsilon^{-2}$ & $\epsilon^{-1}$ & $\epsilon^{0}$ & $\epsilon$ \\
\hline$\left(1^{-}, 2^{-}, 3^{+}, 4^{+}, 5^{+}\right)$ & -5.000000000 & -6.009160505 & 230.9376195 & 702.8219477 \\
$\left(1^{-}, 2^{+}, 3^{-}, 4^{+}, 5^{+}\right)$ & -5.000000000 & -6.009160505 & 1.593323992 & 13.72616843 \\
\hline \hline
\end{tabular}


form which his is left for future work. Even in the absence of analytic results, the timings of our numerical calculation and the availability of master integrals computed in the physical region make it feasible to consider integrating the amplitudes over the physical phase-space and thus start exploring the phenomenology of five-point two-loop gluon processes. This will play a central role in the physics program at the LHC in the near future in particular.

The potential to generalize to other processes is significant, based on our experience with the present computation. The approach is general and requires little spectrumdependent work. In particular, the parametrization of the integrand of the amplitude in terms of master integrals and surface terms, which allows to achieve a full reduction to master integrals, only depends on the kinematics of the process and the power-counting of the theory. The current setup handles five kinematic scales and the generalization to other massless particles is straightforward. Based on the present computation, we also believe that general five-scale processes are attainable and that adding further mass scales will be achievable in the near future. Finally, extensions to nonplanar amplitudes appear well within reach. Given the importance of analytic results for inspiring new methods and for identifying hidden symmetry principles, it will be exciting to learn about the analytic form of the integral coefficients. With the presented methods, we are well set up to explore these directions.

\section{ACKNOWLEDGMENTS}

We thank Z. Bern, M. Jaquier, D. A. Kosower, H. Schönemann and V. Sotnikov for helpful discussions. We thank M. Jaquier for early participation in this work. We thank C. Duhr for the use of his MathEMATICA package PolyLogTools. The work of S. A., F. F. C., and B. P. is supported by the Alexander von Humboldt Foundation, in the framework of the Sofja Kovalevskaja Award 2014, endowed by the German Federal Ministry of Education and Research. H. I.'s work is supported by the Juniorprofessor Program of Ministry of Science, Research and the Arts of the state of Baden-Württemberg, Germany. The work of M.Z. is supported by the U.S. Department of Energy under Award Number DE-SC0009937. This work was performed on the bwUniCluster funded by the Ministry of Science, Research and the Arts Baden-Württemberg and the Universities of the State of Baden-Württemberg, Germany, within the framework program bwHP. This research is supported by the Munich Institute for Astroand Particle Physics (MIAPP) of the DFG cluster of excellence 'Origin and Structure of the Universe'.

\section{APPENDIX: DIVERGENCE STRUCTURE OF TWO-LOOP GLUON AMPLITUDES}

We present results for two-loop gluonic amplitudes with generic helicity configurations. These amplitudes have both infrared and ultraviolet poles. The latter are removed through renormalization of the amplitude. After renormalization, the infrared poles are predicted from lower-order results through a general formula [66]. Here, we briefly summarize the procedure of renormalization and the calculation of the infrared poles for the amplitudes we compute in this paper. Reproducing the expected pole structure described in this section is an important check of our results.

\section{Renormalization of leading-color two-loop gluonic amplitudes}

Renormalization of the amplitude is performed in the $\overline{\mathrm{MS}}$ scheme. It is implemented by replacing the bare coupling by the renormalized one, denoted $\alpha_{S}$, in Eq. (2.1). The bare and renormalized couplings are related through

$\alpha_{0} \mu_{0}^{2 \epsilon} S_{\epsilon}=\alpha_{s} \mu^{2 \epsilon}\left(1-\frac{\beta_{0}}{\epsilon} \frac{\alpha_{s}}{4 \pi}+\left(\frac{\beta_{0}^{2}}{\epsilon^{2}}-\frac{\beta_{1}}{\epsilon}\right)\left(\frac{\alpha_{s}}{4 \pi}\right)^{2}+\mathcal{O}\left(\alpha_{s}^{3}\right)\right)$,

with $S_{\epsilon}=(4 \pi)^{\epsilon} e^{-\epsilon \gamma_{E}}$, where $\gamma_{E}=-\Gamma^{\prime}(1)$ is the EulerMascheroni constant. $\mu_{0}^{2}$ is the scale introduced in dimensional regularization to keep the coupling dimensionless in the QCD Lagrangian, and $\mu^{2}$ is the renormalization scale. In the following we set $\mu^{2}=\mu_{0}^{2}=1$. For purely gluonic amplitudes, the coefficients of the QCD $\beta$ function are

$$
\beta_{0}=\frac{11 N_{C}}{3}, \quad \beta_{1}=\frac{17 N_{C}^{2}}{3} .
$$

The renormalized amplitude is written as

$$
\begin{aligned}
\left.\mathcal{A}_{R}\right|_{\text {leading color }}= & S_{\epsilon}^{-\frac{3}{2}} g_{s}^{3}\left(\mathcal{A}_{R}^{(0)}+\frac{\alpha_{s} N_{C}}{4 \pi} \mathcal{A}_{R}^{(1)}\right. \\
& \left.+\left(\frac{\alpha_{s} N_{C}}{4 \pi}\right)^{2} \mathcal{A}_{R}^{(2)}+\mathcal{O}\left(\alpha_{s}^{3}\right)\right),
\end{aligned}
$$

with the renormalized $\mathcal{A}_{R}^{(i)}$ related to the bare $\mathcal{A}^{(i)}$ as follows:

$$
\begin{aligned}
\mathcal{A}_{R}^{(0)}= & \mathcal{A}^{(0)}, \quad \mathcal{A}_{R}^{(1)}=S_{\epsilon}^{-1} \mathcal{A}^{(1)}-\frac{3}{2} \frac{\beta_{0}}{N_{C} \epsilon} \mathcal{A}^{(0)}, \\
\mathcal{A}_{R}^{(2)}= & S_{\epsilon}^{-2} \mathcal{A}^{(2)}-\frac{5}{2} \frac{\beta_{0}}{N_{C} \epsilon} S_{\epsilon}^{-1} \mathcal{A}^{(1)} \\
& +\left(\frac{15}{8}\left(\frac{\beta_{0}}{N_{C} \epsilon}\right)^{2}-\frac{3}{2} \frac{\beta_{1}}{N_{C}^{2} \epsilon}\right) \mathcal{A}^{(0)} .
\end{aligned}
$$

\section{Infrared behavior}

Renormalization removes all poles of ultraviolet origin. The remaining poles in the renormalized amplitude are of 
infrared origin and can be predicted from the previous orders in the perturbative expansion [66-69]:

$$
\begin{aligned}
& \mathcal{A}_{R}^{(1)}=\mathbf{I}^{(1)}(\epsilon) \mathcal{A}_{R}^{(0)}+\mathcal{O}\left(\epsilon^{0}\right), \\
& \mathcal{A}_{R}^{(2)}=\mathbf{I}^{(2)}(\epsilon) \mathcal{A}_{R}^{(0)}+\mathbf{I}^{(1)}(\epsilon) \mathcal{A}_{R}^{(1)}+\mathcal{O}\left(\epsilon^{0}\right) .
\end{aligned}
$$

In the leading-color approximation, the color structure of loop corrections is the same as that of the leading-order contribution up to a factor of $N_{C}$ that was included in the perturbative expansion parameter, see Eq. (A3). For a $n$ gluon amplitude, the operator $\mathbf{I}^{(1)}$ is then

$$
\mathbf{I}^{(1)}(\epsilon)=-\frac{e^{\gamma_{E} \epsilon}}{\Gamma(1-\epsilon)}\left(\frac{1}{\epsilon^{2}}+\frac{\beta_{0}}{2 N_{C} \epsilon}\right) \sum_{i=1}^{n}\left(-s_{i, i+1}\right)^{-\epsilon},
$$

where $s_{i, i+1}=\left(p_{i}+p_{i+1}\right)^{2}$ with the indices defined cyclically. The operator $\mathbf{I}^{(2)}$ is

$$
\begin{aligned}
\mathbf{I}^{(2)}(\epsilon)= & -\frac{1}{2} \mathbf{I}^{(1)}(\epsilon) \mathbf{I}^{(1)}(\epsilon)-\frac{\beta_{0}}{N_{C} \epsilon} \mathbf{I}^{(1)}(\epsilon) \\
& +\frac{e^{-\gamma_{E} \epsilon} \Gamma(1-2 \epsilon)}{\Gamma(1-\epsilon)}\left(\frac{\beta_{0}}{N_{C} \epsilon}+\frac{67}{9}-\frac{\pi^{2}}{3}\right) \mathbf{I}^{(1)}(2 \epsilon) \\
& +n \frac{e^{\gamma_{E} \epsilon}}{\epsilon \Gamma(1-\epsilon)}\left(\frac{\zeta_{3}}{2}+\frac{5}{12}+\frac{11 \pi^{2}}{144}\right)
\end{aligned}
$$

The poles of the bare amplitudes can be recovered from those of the renormalized amplitude by using Eq. (A4). For amplitudes that are finite at one-loop (such as the all-plus and single-minus helicity configurations) the pole structure of the unrenormalized amplitude is particularly simple because the $1 / \epsilon$ renormalization term in Eq. (A4) matches the $1 / \epsilon$ term in the $\mathbf{I}^{(1)}$ operator [14]. For example, in the all-plus five-gluon case we have

$$
\begin{aligned}
& \mathcal{A}^{(2)}\left(1^{+}, 2^{+}, 3^{+}, 4^{+}, 5^{+}\right) \\
& =-\frac{1}{\epsilon^{2}} \sum_{i=1}^{5}\left(-s_{i, i+1}\right)^{-\epsilon} \mathcal{A}_{R}^{(1)}\left(1^{+}, 2^{+}, 3^{+}, 4^{+}, 5^{+}\right)+\mathcal{O}\left(\epsilon^{0}\right) .
\end{aligned}
$$

In Tables III and IV we present the (normalized) one-loop results needed to perform the checks of the universal pole structure of the two-loop results given in IV. In order to remove unphysical ambiguities related to the normalization of the amplitudes, these are normalized either to the tree amplitude or to the $\epsilon^{0}$ coefficient of the one-loop result if the tree amplitude vanishes. The results in the Tables are obtained with our own implementation of one-loop numerical unitarity. The coefficients are computed in high precision and multiplied by analytic expressions for all one-loop master integrals to obtain the 10 significant digits we present.
[1] J. R. Andersen et al., Les Houches 2013: Physics at TeV Colliders: Standard Model Working Group Report, arXiv:1405.1067.

[2] J. R. Andersen et al., Les Houches 2015: Physics at TeV colliders standard model working group report, arXiv: 1605.04692.

[3] S. Badger, H. Frellesvig, and Y. Zhang, A two-loop fivegluon helicity amplitude in QCD, J. High Energy Phys. 12 (2013) 045.

[4] S. Badger, G. Mogull, A. Ochirov, and D. O'Connell, A complete two-loop, five-gluon helicity amplitude in YangMills theory, J. High Energy Phys. 10 (2015) 064.

[5] Z. Bern, L. J. Dixon, D. C. Dunbar, and D. A. Kosower, One-loop n-point gauge theory amplitudes, unitarity and collinear limits, Nucl. Phys. B425, 217 (1994).

[6] Z. Bern, L. J. Dixon, D. C. Dunbar, and D. A. Kosower, Fusing gauge theory tree amplitudes into loop amplitudes, Nucl. Phys. B435, 59 (1995).

[7] Z. Bern, L. J. Dixon, and D. A. Kosower, One-loop amplitudes for $e^{+} e^{-}$to four partons, Nucl. Phys. B513, 3 (1998).

[8] R. Britto, F. Cachazo, and B. Feng, Generalized unitarity and one-loop amplitudes in $N=4$ super-Yang-Mills, Nucl. Phys. B725, 275 (2005).
[9] G. Ossola, C. G. Papadopoulos, and R. Pittau, Reducing full one-loop amplitudes to scalar integrals at the integrand level, Nucl. Phys. B763, 147 (2007).

[10] P. Mastrolia and G. Ossola, On the Integrand-Reduction Method for Two-Loop Scattering Amplitudes, J. High Energy Phys. 11 (2011) 014.

[11] P. Mastrolia, E. Mirabella, G. Ossola, and T. Peraro, Scattering amplitudes from multivariate polynomial division, Phys. Lett. B 718, 173 (2012).

[12] S. Badger, H. Frellesvig, and Y. Zhang, Hepta-cuts of two-loop scattering amplitudes, J. High Energy Phys. 04 (2012) 055.

[13] Y. Zhang, Integrand-level reduction of loop amplitudes by computational algebraic geometry methods, J. High Energy Phys. 09 (2012) 042.

[14] T. Gehrmann, J. M. Henn, and N. A. Lo Presti, Analytic Form of the Two-Loop Planar Five-Gluon All-Plus-Helicity Amplitude in QCD, Phys. Rev. Lett. 116, 062001 (2016); Erratum, 116, 189903 (2016).

[15] C. G. Papadopoulos, D. Tommasini, and C. Wever, The Pentabox master integrals with the simplified differential equations approach, J. High Energy Phys. 04 (2016) 078.

[16] D. C. Dunbar and W. B. Perkins, Two-loop five-point all plus helicity Yang-Mills amplitude, Phys. Rev. D 93, 085029 (2016). 
[17] D. C. Dunbar, G. R. Jehu, and W. B. Perkins, Two-Loop Six Gluon All Plus Helicity Amplitude, Phys. Rev. Lett. 117, 061602 (2016).

[18] D. C. Dunbar, J. H. Godwin, G. R. Jehu, and W. B. Perkins, Analytic all-plus gluon amplitudes in QCD, Phys. Rev. D 96, 116013 (2017).

[19] S. Badger, C. Brønnum-Hansen, H. B. Hartanto, and T. Peraro, A First Look at Two-Loop Five-Gluon Scattering in QCD, Phys. Rev. Lett. 120, 092001 (2018).

[20] R. K. Ellis, W. T. Giele, and Z. Kunszt, A numerical unitarity formalism for evaluating one-loop amplitudes, J. High Energy Phys. 03 (2008) 003.

[21] W. T. Giele, Z. Kunszt, and K. Melnikov, Full one-loop amplitudes from tree amplitudes, J. High Energy Phys. 04 (2008) 049.

[22] C. F. Berger, Z. Bern, L. J. Dixon, F. Febres Cordero, D. Forde, H. Ita, D. A. Kosower, and D. Maitre, An automated implementation of on-shell methods for one-loop amplitudes, Phys. Rev. D 78, 036003 (2008).

[23] H. Ita, Two-loop integrand decomposition into master integrals and surface terms, Phys. Rev. D 94, 116015 (2016).

[24] S. Abreu, F. F. Cordero, H. Ita, M. Jaquier, and B. Page, Subleading poles in the numerical unitarity method at two loops, Phys. Rev. D 95, 096011 (2017).

[25] S. Abreu, F. F. Cordero, H. Ita, M. Jaquier, B. Page, and M. Zeng, Two-Loop Four-Gluon Amplitudes from Numerical Unitarity, Phys. Rev. Lett. 119, 142001 (2017).

[26] K. G. Chetyrkin and F. V. Tkachov, Integration by parts: The algorithm to calculate $\beta$ functions in 4 loops, Nucl. Phys. B192, 159 (1981).

[27] S. Laporta, High-precision calculation of multi-loop Feynman integrals by difference equations, Int. J. Mod. Phys. A 15, 5087 (2000).

[28] S. Laporta and E. Remiddi, The analytical value of the electron $(g-2)$ at order $\alpha^{3}$ in QED, Phys. Lett. B 379, 283 (1996).

[29] S. Abreu et al. (to be published).

[30] A. von Manteuffel and R. M. Schabinger, A novel approach to integration by parts reduction, Phys. Lett. B 744, 101 (2015).

[31] T. Peraro, Scattering amplitudes over finite fields and multivariate functional reconstruction, J. High Energy Phys. 12 (2016) 030.

[32] G. 't Hooft and M. J. G. Veltman, Regularization and renormalization of gauge fields, Nucl. Phys. B44, 189 (1972).

[33] Z. Bern and D. A. Kosower, The computation of loop amplitudes in gauge theories, Nucl. Phys. B379, 451 (1992).

[34] Z. Bern, A. De Freitas, L. J. Dixon, and H. L. Wong, Supersymmetric regularization, two loop QCD amplitudes and coupling shifts, Phys. Rev. D 66, 085002 (2002).

[35] J. Gluza, K. Kajda, and D. A. Kosower, Towards a basis for planar two-loop integrals, Phys. Rev. D 83, 045012 (2011).

[36] R. M. Schabinger, A new algorithm for the generation of unitarity-compatible integration by parts relations, J. High Energy Phys. 01 (2012) 077.

[37] K. J. Larsen and Y. Zhang, Integration-by-parts reductions from unitarity cuts and algebraic geometry, Phys. Rev. D 93, 041701 (2016).
[38] C. Anastasiou and A. Lazopoulos, Automatic integral reduction for higher order perturbative calculations, J. High Energy Phys. 07 (2004) 046.

[39] A. V. Smirnov, Algorithm FIRE-Feynman Integral REduction, J. High Energy Phys. 10 (2008) 107.

[40] A. V. Smirnov, FIRE5: a $\mathrm{C}++$ implementation of Feynman Integral REduction, Comput. Phys. Commun. 189, 182 (2015).

[41] C. Studerus, Reduze-Feynman integral reduction in $\mathrm{C}++$, Comput. Phys. Commun. 181, 1293 (2010).

[42] A. von Manteuffel and C. Studerus, Reduze 2-Distributed Feynman Integral Reduction, arXiv:1201.4330.

[43] R. N. Lee, LiteRed 1.4: a powerful tool for reduction of multiloop integrals, J. Phys. Conf. Ser. 523, 012059 (2014).

[44] P. Maierhoefer, J. Usovitsch, and P. Uwer, Kira-A Feynman Integral Reduction Program, Comput. Phys. Commun. 230, 99 (2018).

[45] A. Georgoudis, K. J. Larsen, and Y. Zhang, Azurite: An algebraic geometry based package for finding bases of loop integrals, Comput. Phys. Commun. 221, 203 (2017).

[46] H. Ita, Towards a numerical unitarity approach for two-loop amplitudes in QCD, Proc. Sci., LL2016 (2016) 080.

[47] Y. Zhang, Lecture notes on multi-loop integral reduction and applied algebraic geometry, arXiv:1612.02249.

[48] Z. Bern, M. Enciso, H. Ita, and M. Zeng, Dual conformal symmetry, integration-by-parts reduction, differential equations and the nonplanar sector, Phys. Rev. D 96, 096017 (2017).

[49] W. Decker, G.-M. Greuel, G. Pfister, and H. Schönemann, SINGULAR 4-1-0 - A computer algebra system for polynomial computations, http://www.singular.uni-kl.de (2016).

[50] A. Hodges, Eliminating spurious poles from gauge-theoretic amplitudes, J. High Energy Phys. 05 (2013) 135.

[51] F. A. Berends and W. T. Giele, Recursive calculations for processes with n gluons, Nucl. Phys. B306, 759 (1988).

[52] R. K. Ellis, Z. Kunszt, K. Melnikov, and G. Zanderighi, One-loop calculations in quantum field theory: from Feynman diagrams to unitarity cuts, Phys. Rep. 518, 141 (2012).

[53] P. Nogueira, Automatic Feynman graph generation, J. Comput. Phys. 105, 279 (1993).

[54] A. Ochirov and B. Page, Full colour for loop amplitudes in Yang-Mills theory, J. High Energy Phys. 02 (2017) 100.

[55] M. Brickenstein, Slimgb: Gröbner bases with slim polynomials, Revista Matemática Complutense 23, 453 (2010).

[56] J. A. M. Vermaseren, New features of FORM, arXiv: math-ph/0010025.

[57] J. Kuipers, T. Ueda, J. A. M. Vermaseren, and J. Vollinga, FORM version 4.0, Comput. Phys. Commun. 184, 1453 (2013).

[58] B. Ruijl, A. Plaat, J. Vermaseren, and J. van den Herik, Why local search excels in expression simplification, arXiv: 1409.5223.

[59] M. Abramowitz and I. A Stegun, Handbook of Mathematical Functions: With Formulas, Graphs, and Mathematical Tables, Vol. 55 (Courier Corporation, Massachusetts, 1964).

[60] T. Gautier, J.-L. Roch, and G. Villard, Givaro, C++ library for arithmetic and algebraic computations, http://givaro .forge.imag.fr. 
[61] P. Barrett, Implementing the rivest shamir and adleman public key encryption algorithm on a standard digital signal processor, in Advances in Cryptology-CRYPTO'86: Proceedings, edited by A. M. Odlyzko (Springer, Berlin, 1987), p. 311.

[62] J. van der Hoeven, G. Lecerf, and G. Quintin, Modular SIMD arithmetic in mathemagix, CoRR abs/1407.3383, arXiv:1407.3383.

[63] T. Gehrmann and E. Remiddi, Two-loop master integrals for $\gamma * \rightarrow 3$ jets: The planar topologies, Nucl. Phys. B601, 248 (2001).

[64] J. Vollinga and S. Weinzierl, Numerical evaluation of multiple polylogarithms, Comput. Phys. Commun. 167, 177 (2005).
[65] A. V. Smirnov, FIESTA4: Optimized Feynman integral calculations with GPU support, Comput. Phys. Commun. 204, 189 (2016).

[66] S. Catani, The singular behavior of QCD amplitudes at two loop order, Phys. Lett. B 427, 161 (1998).

[67] G. F. Sterman and M. E. Tejeda-Yeomans, Multi-loop amplitudes and resummation, Phys. Lett. B 552, 48 (2003).

[68] T. Becher and M. Neubert, Infrared Singularities of Scattering Amplitudes in Perturbative QCD, Phys. Rev. Lett. 102, 162001 (2009); Erratum, 111, 199905 (2013).

[69] E. Gardi and L. Magnea, Factorization constraints for soft anomalous dimensions in QCD scattering amplitudes, J. High Energy Phys. 03 (2009) 079. 